\title{
Psychometric properties and measurement invariance of the Polish version of the dyadic coping inventory
}

\author{
Anna M. Wendołowska ${ }^{1}$ (D) $\cdot$ Dorota Czyżowska $^{1}$ (D) - Guy Bodenmann ${ }^{2}$ (i) \\ Published online: 31 January 2020 \\ (C) The Author(s) 2020
}

\begin{abstract}
Recently, there has been growing interest in stress and coping strategies from a relational perspective. Researchers recognize the importance of a partner's coping strategy in terms of relationship satisfaction and a relational sense of well-being. The aim of the article is to present the results of work on the Polish validation of the Dyadic Coping Inventory (DCI), which was developed by Bodenmann et al. (2008). Dyadic coping is a multidimensional construct which consists of stress communication by partners and four components of dyadic coping behavior: supporting, delegated, negative and common behavior. The DCI examines the quality of communication between partners and the level of dyadic coping with stress. The DCI was tested based on a study of 550 people ( 275 couples) aged between 20 and 72 . The conducted analyses were aimed at checking the factor structure and psychometric properties of the Polish version of the DCI. Measurement invariance (MI) was also tested across genders on a Polish sample and across cultures on Polish, Swiss and Romanian samples. After rejection of three pairs of items, factor analysis of the DCI revealed a 5-factor structure with respect to a partner's own dyadic coping and their partner's dyadic coping and a 2factor structure with respect to common dyadic coping. MI results confirmed the criteria for configural, metric and full scalar invariance across genders and configural, metric and partial scalar invariance across cultures. The Polish version of DCI has satisfactory psychometric characteristics and can be used to assess the dyadic coping of people in romantic relationships; also, it can be used in cross-cultural studies.
\end{abstract}

Keywords Dyadic coping inventory; psychometrics; couple $\cdot$ Close relationships $\cdot$ Social support $\cdot$ Coping

\section{Introduction}

Over the years, the stress and coping construct was considered at an individual level (Endler and Parker 1994; Lazarus and Folkman 1984). At the end of the twentieth century, the first theories emerged that relate to coping with stress at the relational level (Pearlin and Schooler 1978; Coyne and Smith 1991; DeLongis and O'Brien 1990; Revenson et al. 2003; Bodenmann 2005). The concept of Pearlin and Schooler (1978) assumes that partners in the context of a relationship

Special thanks are due to Dr Petruta Rusu for substantive support and sharing of the database

Anna M. Wendołowska anna.wendolowska@doctoral.uj.edu.pl

1 Institute of Psychology, Jagiellonian University, 6 Ingardena Street, 30-060 Krakow, Poland

2 Psychologisches Institut, Klinische Psychologie Kinder/Jugendliche \& Paare/Familien, Binzmühlestrasse 14/23,

CH-8050 Zürich, Switzerland apply individual strategies that are developed and enriched regardless of relational factors. The results of their research indicate that individual strategies for coping with stress are most effective in interpersonal problem situations and are least effective in the case of external stress (Pearlin and Schooler 1978). The latest theories about stress and coping confirm that individual stress often becomes dyadic stress that affects both partners (see: Martos et al. 2019). Stress can cause a crisis in a relationship that can lead to its breakup or may become a driver of potential growth (Story and Bradbury 2004). The way in which a partner engages in the process of coping with the other partner's stress is of great importance not only for the quality of the relationship, but also this can ultimately determine the effectiveness of the strategies used (see: Chow et al. 2014). Revenson et al. (2003) focus on the congruency between the individual efforts of partners; Coyne and Fiske (1992) emphasize the importance of the support provided by one partner in the coping process of the other, which in consequence improves relational functioning and leads to a betterquality relationship. Empathic response in dyadic coping is potentially important in solving interpersonal problems 
(DeLongis and O'Brien 1990) and is also related to better relational adjustment; it can also be an adaptive way of coping with daily stress (Bokhari 2006).

The systemic-transactional model (STM; Bodenmann 2005) defines dyadic coping as a circular interpersonal process involving both individual and joint appraisals, common goals and resources. Dyadic coping involves not only support from the partner, but also stress communication and partners' joint coping efforts as a response to stress (Bodenmann 1997). The Dyadic Coping Inventory (DCI; Bodenmann 2008) is a widely used self-report instrument designed to measure dyadic coping between partners (e.g., Austin and Falconier 2013; Heinrichs et al. 2011; Meuwly et al. 2012). The DCI measures partners' behaviors when one or both partners experience stress. Partners report on their own behaviors and their perception of their partner's behaviors, as well as how they respond jointly in the face of a common stressor.

Studies confirm a significant relationship between positive dyadic coping and higher relational satisfaction and the quality of relationships (Bodenmann et al. 2006, 2007 2010; Falconier et al. 2013b; Iafrate et al. 2012; Kardatzke 2009; Randall and Bodenmann 2017). Studies on dyadic coping carried out on clinical populations showed that positive dyadic coping had the potential to reduce depressive symptoms (Bodenmann et al. 2008; Gabriel et al. 2016; Johnson et al. 2017) and strengthen the quality of the relationship (Beach et al. 2014). Breast cancer patients and partners who presented more common dyadic coping declared higher relational satisfaction (Badr et al. 2010) and negative behaviors were associated with depressed mood for both patients and their partners (Rottmann et al. 2015). A study of another population of patients with chronic obstructive pulmonary disease (Vaske et al. 2015) showed that the patients' positive dyadic coping was strongly associated with higher quality of life for their partners. Based on the aforementioned results of previous studies, we can expect that our study on the Polish population will confirm the significant relationship between dyadic coping and relational quality and satisfaction.

\section{The Systemic-Transactional Model (STM) of Dyadic Coping}

The concept of dyadic coping was created in the 1990s. Bodenmann (2005) expanded the transactional stress theory by Lazarus and Folkman (1984) into a systemic perspective. Dyadic coping is the complex behavioral-emotional response system of both partners in response to stress experienced by one or both of them. In the case of people living in close relationships, it is not possible to perceive coping strategies without considering the role of the partner in the coping process. Verbal or non-verbal signals that communicate the stress experienced by one partner affect and induce reactions in the other. The manner of receiving, interpreting and decoding signals sent by the partner, and the form of response or lack thereof due to lack of competence or motivation all have an impact on the evaluations, behaviors and decisions taken by the person experiencing stress. Effective personal and dyadic adaptation means a return to the state before the appearance of the stressor in which both partners find a new homeostasis, well-being and satisfaction (Bodenmann 2005). Dyadic coping is aimed not only at supporting the partner, but at the same time reducing the other partner's stress level. If not effectively reduced, stress affecting one partner also affects the other person in the relationship, so it is in the interest of both partners to support each other for the sake of their own well-being, satisfaction and the stability of the relationship.

Dyadic coping (DC) is a multidimensional concept that comprises (a) stress communication and (b) four components of dyadic coping, which are called supportive DC, delegated DC, negative DC and common DC. Stress Communication (SC) refers to verbal and nonverbal stress signals sent by one partner to the other. Supportive DC (SDC) means assisting the partner in everyday duties, finding solutions or reframing problems, as well as helping with the regulation of emotions by showing understanding and empathy and expressing solidarity with the partner. Delegated DC (DDC) occurs when one partner takes over the other's duties to minimize or reduce his or her burden. This type of support is most often found in situations requiring a problem-oriented action (Bodenmann 2005). Negative DC (NDC) comprises negative behaviors such as hostile, ambivalent and superficial ones. Hostile behaviors include insulting remarks regarding the partner's stress or individual coping, evasion, sarcasm, mockery, manifestation of obvious lack of interest and minimizing the importance of stress experienced by the partner. The partner provides support but does so in an undesirable, denigrating or humiliating way, presenting hostility often on a non-verbal or para-verbal level. Ambivalent behaviors are defined as providing support with reluctance or the belief that it is unnecessary to get involved in the problem and that the partner should be able to get along without support. Superficial behavior is expressed in support characterized by insincerity, lack of attention and true empathy towards the partner. Common DC (CDC) encompasses coping attempts involving both partners in a symmetrical or complementary way, focusing on solving the problem or reducing negative emotions, using strategies to jointly search for solutions or information, sharing feelings, confirming commitment and seeking common forms of relaxation (Bodenmann 1995; Falconier et al. 2016).

The main function of dyadic coping is to reduce the stress level of both partners. Both partners should be motivated to help each other in stressful situations and work together in the process of coping with stress because the well-being and satisfaction of one partner depends on the well-being and satisfaction of the other. Additionally, DC strengthens the 
relationship by increasing and fostering mutual understanding, trust, and feelings of we-ness and intimacy (Bodenmann 2005).

\section{The Factorial Structure of the Dyadic Coping Inventory (DCI)}

Based on the systemic-transactional model of dyadic coping, the DCI was developed for the assessment of the different forms of dyadic coping: SC, SDC, DDC, NDC, and CDC (Bodenmann 2008). In the original German version of the DCI (Bodenmann 2008) and in studies on Italian, French and Spanish samples (Ledermann et al. 2010), exploratory factor analyses showed the four-factor structure of DC by Self and Partner (SC, SDC, DDC, and NDC) and the onefactor structure of CDC. Confirmatory factor analysis showed a slightly different structure. In the Italian validation study (Donato et al. 2009), a five-factor solution was proposed for the assessment DC by Self and by Partner (SC, emotionfocused SDC, problem-focused SDC, DDC, and NDC) and a three-factor solution for CDC (problem-seeking CDC, proximity-seeking $\mathrm{CDC}$, and relaxation $\mathrm{CDC}$ ). The English validation of the DCI in the Canadian population (Levesque et al. 2014) confirmed the five-factor structure of DC by Self and by Partner (SC, emotion-focused SDC, problem-focused SDC, DDC, and NDC) and the one-factor structure of CDC. Validation studies in Spanish, Persian, Romanian, Portuguese, and Chinese (Falconier et al. 2013a; Fallahchai et al. 2017; Rusu et al. 2016; Vedes et al. 2013; Xu et al. 2016) showed the same five-factor solution for the DC by Self and Partner and a two-factor solution for CDC (problem-focused CDC and emotion-focused CDC). In all these studies the DCI comprised very good validity. Furthermore Xu et al. (2016) compared $5+2$-factor and $4+1$-factor models within three cultural groups. The results confirmed that the five-factor solution for DC by Self and by Partner and the two-factor solution for CDC appeared to the most appropriate in terms of model fit and consistency across the analyses, hence Polish validation studies was decided to test 5 -factor structure of DC by Self and by Partner and 2-factor solution for CDC.

\section{The Purpose of the Study}

The main purpose of this study was to validate the Polish version of the Dyadic Coping Inventory by examining its psychometric properties in a Polish sample of heterosexual couples. The study aims to confirm the factor-based structure of the DCI separately for each partner in order to check the homogeneity of the assessment tool. The Polish version of the DCI is based on the five-factor structure of DC by Self and by Partner and the two-factor structure of CDC. Confirmation of the factor structure of the DCI will also confirm its theoretical validity.
Another aim of this study was to confirm the criterion validity of the DCI. Significant correlations were expected 1) between DC by Self (stress communication (SC), emotionfocused supportive DC (ESDC), problem-focused supportive DC (PSDC), delegated DC (DDC)) and aggregated DC by Self, combining 5 DC by Self subscales (SC, ESDC, PSDC, DDC, NDC); 2) between DC by Self (SC, ESDC, PSDC, DDC, NDC) and aggregated DC by Partner, combining 5 DC by Partner subscales (e.g. Fallahchai et al. 2017); and 3) between DC by Self subscales (SC, ESDC, PSDC, DDC, NDC) and corresponding DC by Partner subscales (SC, ESDC, PSDC, DDC, NDC) (e.g. Randal et al. 2016).

The additional purpose of the study was to test measurement invariance (MI) across gender and culture, which is crucial to demonstrate that the items of the instrument measure the same construct (Vandenberg and Lance 2000). Thus far, MI across genders and cultures was tested in the English, Romanian and Chinese DCI validation studies (Randall et al. 2016; Rusu et al. 2016; Xu et al. 2016) and also across genders only, in Portuguese DCI validation studies (Vedes et al. 2013), for the five- and two-factor solution, which further substantiate the selection of the 5- + 2-factor DCI model for Polish validation.

Assuming that all three types of MI, configural, metric, and scalar, can be confirmed, this would provide evidence that items and constructs of the DCI have similar meaning and are interpreted across groups the same way. Also comparing the results of men and women on dyadic coping scales will determine the true significant gender differences, which is also relevant for coping research (Bodenmann et al. 2006).

\section{Method}

Following Beaton and colleagues' suggestions (Beaton et al. 2000), the original German version of the DCI was translated into Polish independently by two native Polish professionals who were proficient in German. Inappropriate choices were identified and resolved for each item separately through discussions among the judges and researchers responsible for adapting the instrument. The two translated versions were synthesized into one single version that was then evaluated by the research team (Gudmundsson 2009) and backtranslated into German by a professional translator with a psychological education background. In the German version, 4 sentences were clarified by the original author, Prof. Dr. Bodenmann, in order to maintain conceptual equivalence (Beaton et al. 2000). For example, in the sentence "Ich sage meiner Partnerin, dass ich ihre Unterstützung, Rat und Hilfe schätze "(I openly tell my partner how I feel and that I appreciate her support), the word "schätzen" (appreciate) was corrected to "benötigen/brauchen "(need/expect) to highlight not appreciation but communication of need. After final 
evaluation by the original author, the instrument's adaptation was completed and statistical analyses were performed.

All procedures performed in studies involving human participants were in accordance with the ethical standards of the institutional research committee (the Ethics Committee at the Institute of Psychology, Jagiellonian University; KE/01/ 102018) and with the 1964 Helsinki declaration and its later amendments or comparable ethical standards. Informed consent was obtained from all individual participants in the study.

\section{Participants}

The study involved participants from several counties in Poland who were in heterosexual relationships. Participants were recruited in various places using traditional methods such as leaflets, advertisements and email. The qualifying criterion for participation in the study was being in a formal or informal heterosexual relationship for a minimum of 12 months. Those interested reported by email their consent to participate in the study. They were informed about the confidentiality and scientific nature of the research. They individually received questionnaires and returned them completed at the agreed place and time; full confidentiality of the data was maintained. As incentives some randomly assigned subjects received cinema tickets.

Of the approximately 2000 subjects interested in the study, 275 couples $(n=550$ individuals) returned completed questionnaires: $43.8 \%$ of respondents were aged 41-60 $(n=$ $241) ; 37.64 \%$ were aged $26-40(n=207) ; 15.27 \%$ were aged $18-25(n=84)$. The vast majority of respondents $(68.91 \%)$ were married $(n=379)$ and $31.64 \%(n=174)$ were cohabiting. $24.19 \%$ of respondents declared a relationship duration of $6-10$ years, $23.81 \%$ in the range $1-5$ years, and $23.67 \%$ in the range 11-20 years. Almost $72 \%$ of participants had at least one child $(n=393)$. Over $71 \%$ of respondents reported higher education $(n=394), 23.64 \%$ reported secondary education $(n=130)$, and slightly above $3 \%$ reported primary, junior high school and vocational education $(n=19)$. Most respondents $(64.72 \%)$ reported a good to very good financial situation $(n=$ $364)$, almost $33 \%(n=181)$ assessed their financial situation as rather good, only $2 \%(n=12)$ assessed their financial situation as bad. Most of the subjects $(83.82 \%)$ were professionally active $(n=361), 12.55 \%$ were students, $2.55 \%$ were retired and only $1.09 \%$ were unemployed.

\section{Swiss and Romanian Samples}

To evaluate measurement invariance across cultures, the data of Swiss and Romanian samples were used. The Swiss sample was selected to test MI across Polish and Swiss couples due to the fact that the DCI was originally developed in Switzerland. Testing MI across Polish and Romanian samples was interesting for us as Romania is the only representative that validated the DCI and that has a post-communist, Eastern European country with a similar political, social and historical background as Poland. The Swiss sample included 368 heterosexual couples ( $n=736$ individuals): men's mean age was 49.3 years $(S D=18.3)$, women's mean age was 47.3 years $(S D=18.3)$ and average relationship duration was $21(S D=$ 17.88). The Romanian sample included 510 married heterosexual couples ( $n=1.020$ individuals): men's mean age $=$ 39.0 years $(S D=9.3)$, women's mean age $=36.1$ years $(S D=9.2)$, average marriage duration was 13.0 years $(S D=$ 9.1). The Romanian sample was used in the Romanian validation study of the DCI (Rusu et al. 2016) using the English language version of the instrument. The Swiss sample was used in a longitudinal project that started 8 years ago entitled "Impact of stress on relationship development of couples and children: A longitudinal approach on dyadic development across the lifespan". There are three official languages in Switzerland: German, French and Italian. In the Swiss sample, the German language version of the DCI was used, on the basis of which the Polish-language version was created.

\section{Measures}

\section{The Dyadic Coping Inventory DCI}

(Bodenmann 2008) is a 37-item questionnaire that assesses how couples cope with stress. Respondents complete the inventory by marking answers on a 5-point scale ranging from $1=$ very rarely to $5=$ very often, assessing the following:

1) dyadic coping (DC) by Self, regarding own behaviors: a) stress communication (SC), e.g., I openly tell my partner how I feel and when I need support; b) emotion-focused supportive DC (ESDC), e.g., I show my partner compassion and understanding; c) problem-focused supportive DC (PSDC), e.g., I help my partner to see stressful situations in a different light; d) delegated DC (DDC), e.g., I take on things that my partner would normally do in order to help him/her out; d) negative DC (NDC), e.g. I do not take my partner's problems seriously.

2) DC by Partner, regarding participants' perceptions of partner's behaviors: SC, SDC, DDC, NDC.

3) common DC, e.g., both of us try to face problems together and find specific solutions together.

Two aggregated scales, DC by Self and DC by Partner, are formed by combining 5 subscales: SC, ESDC, PSDC, DDC and NDC. Item contents and the number of items per subscale are equivalent across Self and Partner. The same behavior is evaluated in relation to a partner's own behavior (DC by Self) and then in relation to the other partner (DC by Partner): e.g., I show my partner compassion and understanding (ESDC by Self); my partner shows empathy and understanding to me (ESDC by Partner). In addition, the DCI contains two items (36 and 37), by means of which the respondents evaluate their satisfaction and the effectiveness of dyadic coping. The total 
score of dyadic coping is the sum of all items 1 to 35 after reverse coding is applied to the negative behavior scale items. Items 36 and 37 are not included in the overall score. A total score lower than 111 means low DC, a score greater than 145 signifies higher DC than usual. The scale shows good internal consistency in different languages (Bodenmann 2008).

\section{The Partner Relationship Questionnaire}

PFB developed by Hahlweg (1996) and validated by Janicka (2008) is a 30-item questionnaire for assessing the quality of close relationships. It consists of three subscales: quarrelling (Q: e.g., he ridicules my statements), tenderness (T: e.g., he hugs me) and communication (C: e.g., he tells me openly about his thoughts and feelings). In research in Polish samples, the internal consistency was Cronbach's $\alpha=.88$ : .85 for the $\mathrm{C}$ dimension, .88 for the $\mathrm{T}$ dimension and .90 for the $\mathrm{Q}$ dimension. The questionnaire can be used to diagnose problems in inter-partner relations. The PFB was created for diagnostic purposes to qualitatively distinguish well-functioning couples from those in need of therapy.

\section{The Married and Cohabiting Couples' Communication Questionnaire}

(KKMiP) (Kaźmierczak and Plopa 2012) is a self-report questionnaire: it contains 30 items that relate to a partner's own behavior and 30 items in which the respondent evaluates the partner's behavior on a scale from $1=$ never to $5=$ always. It contains three subscales to measure three communication styles used by Self and Partner. Two of the subscales rate positive behaviors: support (S: e.g., I give my partner psychological, emotional and spiritual support) and involvement (I: e.g., I make small, pleasant surprises for my partner). One of the subscales rates negative behavior: depreciation (D: e.g., I criticize my partner). The sum of the results of the three subscales (the result of the depreciation subscale is obtained by subtracting the raw score from 66 ) gives the total result. The reliability of the KKMiP is $\alpha=.89$.

\section{Analysis Strategies}

The main goal of the research is to a) assess the factorial structure of the Polish version of the Dyadic Coping Inventory using Exploratory Factor Analysis and Confirmatory Factor Analysis; b) analyze convergent validity to confirm a significant relationship between the DCI and the questionnaires investigating related constructs; c) analyze the criterion validity of the DCI by confirming the direction and strength of relationships between individual subscales and aggregated scales (combined DC by Self and DC by Partner scales); d) test measurement invariance (MI) in order to evaluate whether the DCI items measure the same construct across genders and cultures.
CFA and MI analyses were carried out using the lavaan package (Rosseel 2012) in the R environment ( $R$ Core Team 2016). All other analyses were performed using the IBM SPSS Statistics 24 statistical package.

\section{Results}

\section{Confirmatory Factor Analysis of the DCI}

In order to check the structure of the DCI, a 5-factor and 2factor model were tested by confirmatory factor analysis. The tested model included positions for the sample of 550 couples: women $(n=275)$ and men $(n=275)$. In the tested model, items $1-35$ of the DCI were included. Fit indices did not show a good fit of the data for the 5-factor model for men or women for the aggregated scales DC by Self and DC by Partner (Table 1). After rechecking the structure and value of factor loads of all items, it turned out that all regression paths were significant except item 15 (.079 for men and .054 for women) in NDC by Partner. Also, factor loadings for item 15 were very low (.125 for men and .141 for women) and related not only to negative but also to positive factors and DC subscales. After removing item 15 from the NDC by Partner and corresponding item 26 from the NDC by Self, it turned out that the fit indices improved, but not significantly. Further inspection of the results indicated that items 17 and 18 from SC by Partner and corresponding item 2 and 3 from SC by Self scale showed lower factor loadings and residual correlations mainly with ESDC and PSDC. These pairs of items were excluded from the SC scale, which resulted in good fit indices, according to Hu and Bentler's (1999) cutoff criteria (Table 2).

In the case of the 2-factor model for women, fit indices show a very good fit of the data. The TLI index in the 5-factor model for men and RMSEA for men in the 2-factor model achieved slightly weaker values (Table 2). The obtained beta values of regression coefficients, which determine factor loads for individual items (Fig.1), make it possible to conclude that most items have good properties. Only item 4/19 has slightly lower beta values, but it is still at a satisfactory level. It can be concluded that the $5+2$-factor model is well suited to the data, although, as in many other previous validation studies (Ledermann et al. 2010; Falcontier et al. 2012; Levesque et al. 2014; Randal et al. 2016; $\mathrm{Xu}$ et al. 2016; Rusu et al. 2016), some items had to be removed from the original version of the DCI.

\section{Descriptive Statistics of the DCI}

The result of the $t$-test (Table 3) shows that the gender differences of the studied behaviors exist only in the stress communication and delegated DC scales by Self and by Partner. In the remaining subscales and the entire scale, no gender differences are observed. 
Table 1 Goodness of Fit Indices for the CFA of DC by Self, by Partner, and Common DC before modifications

\begin{tabular}{cllccccccc}
\hline & & \multicolumn{1}{c}{$\chi^{2}$} & $d f$ & $p$ & CFI & TLI & RMSEA & SRMR \\
\hline \multirow{2}{*}{ Women } & 5-factor & DC by Self & 257.89 & 80 & $<0.001$ & 0.83 & 0.78 & 0.09 & 0.09 \\
& & DC by Partner & 274.52 & 80 & $<0.001$ & 0.90 & 0.86 & 0.09 & 0.11 \\
& \multirow{4}{*}{ 2-factor } & Common DC & 274.53 & 4 & 0.12 & 1.00 & 0.99 & 0.05 & 0.02 \\
& \multirow{2}{*}{ 5-factor } & DC by Self & 269.80 & 80 & $<0.001$ & 0.89 & 0.85 & 0.09 & 0.08 \\
& \multirow{2}{*}{ 2-factor } & DC by Partner & 270.65 & 80 & $<0.001$ & 0.86 & 0.82 & 0.09 & 1.00 \\
& Common DC & 12.91 & 4 & 0.012 & 0.99 & 0.97 & 0.09 & 0.03 \\
\hline
\end{tabular}

$D C$ dyadic coping

\section{Reliability of the DCl}

Cronbach's alpha was used to determine the internal consistency between the DCI subscales (Table 3). Based on the results, an alpha coefficient of .86 for the entire scale was estimated. Acceptable and very good reliability at the level from .70 to .92 was obtained for most aggregated scales. The exceptions are the NDC and SC subscales, which present lower reliability. Given that most of the DCI subscales are very short, internal consistency should be assessed as high.

The results of Spearman's correlation analysis confirmed the direction and strength of relationships between the subscales and aggregated DCI scales (Table 4). Several coefficients reached high values, thus indicating a strong association of the DCI scales with the dyadic coping construct. The exception is DDC by Self in women, which corelates significantly only with the SC by Partner scale, and NDC by Self does not correlate significantly with PSDC by Self in men and with $\mathrm{SC}$ and DDC by Self in women.

\section{Convergent Validity}

In order to analyze the convergent validity of the DCI, correlation analyses were carried out to confirm that there is a significant relationship between the PFB and the KKMiP questionnaires investigating related constructs (Campbell and Fiske 1959). The results of the Spearman correlation analysis confirmed the direction and strength of the relationships between the scales of the DCI and the PFB and the KKMiP (Table 5).
The results of the analysis of the relationship between dyadic coping and the quality of relationships and partner communication show that the scale of positive behaviors of the DCI are strongly correlated with the relational quality measured with the PFB (the communication (C) and tenderness (T) scales), and positive partner's communication, assessed with the KKMiP (the support (S) and the involvement (Inv) scales). The NDC subscale is strongly positively correlated with the negative PFB quarrelling (Q) scale and negative partner communication, measured using the KKMiP depreciation (D) scale. Differences between the results of men and women are observed. Correlations between DDC by Self in women and the tenderness $(\mathrm{T})$ and the quarrelling $(\mathrm{Q})$ scales, depreciation (D) by Self and depreciation (D) by Partner, support (S) by Partner and involvement (I) by Partner appear to be insignificant. Correlation between the PFB quarrelling (Q) scale and the DC by Self scales (SCS, SCP, PSDC and DDC) turn out to be statistically insignificant for women. There is also no statistically significant relationship between NDC by Partner and the KKMiP involvement (I) by Self in women. The strongest correlations occur between ESDC by Partner and perceived support (S) and involvement (I) by Partner in both women and men and PCDC and communication $(\mathrm{C})$ in men.

\section{Measurement Invariance}

Measurement Invariance was tested for the 5-factor and 2factor models to determine whether the DCI constructs were equivalent to configural, metric, and scalar invariance levels across cultures and genders. Five models were fitted with

Table 2 Goodness of Fit Indices for the CFA of DC by Self, by Partner and Common DC after modifications

\begin{tabular}{|c|c|c|c|c|c|c|c|c|c|c|}
\hline & & & $x^{2}$ & $d f$ & $p$ & CFI & TLI & RMSEA & CIs for RMSEA & SRMR \\
\hline \multirow[t]{3}{*}{ Women } & 5-factor & DC by Self & 139.67 & 44 & $<.001$ & .973 & .960 & .059 & $.073-.106$ & .049 \\
\hline & & DC by Partner & 89.06 & 44 & $<.001$ & .973 & .960 & .061 & $.043-.079$ & .047 \\
\hline & 2 -factor & Common DC & 7.23 & 4 & .124 & .996 & .990 & .054 & $.000-.116$ & .018 \\
\hline \multirow[t]{3}{*}{ Men } & 5-factor & DC by Self & 97.67 & 44 & $<.001$ & .960 & .940 & .067 & $.049-.084$ & .044 \\
\hline & & DC by Partner & 119.89 & 44 & $<.001$ & .937 & .906 & .079 & $.062-.096$ & .044 \\
\hline & 2 -factor & Common DC & 12.91 & 4 & .012 & .989 & .972 & .090 & $.038-.147$ & .027 \\
\hline
\end{tabular}

$D C$ dyadic coping 


\section{Item self/partner Women self/partner \\ Men self/partner}
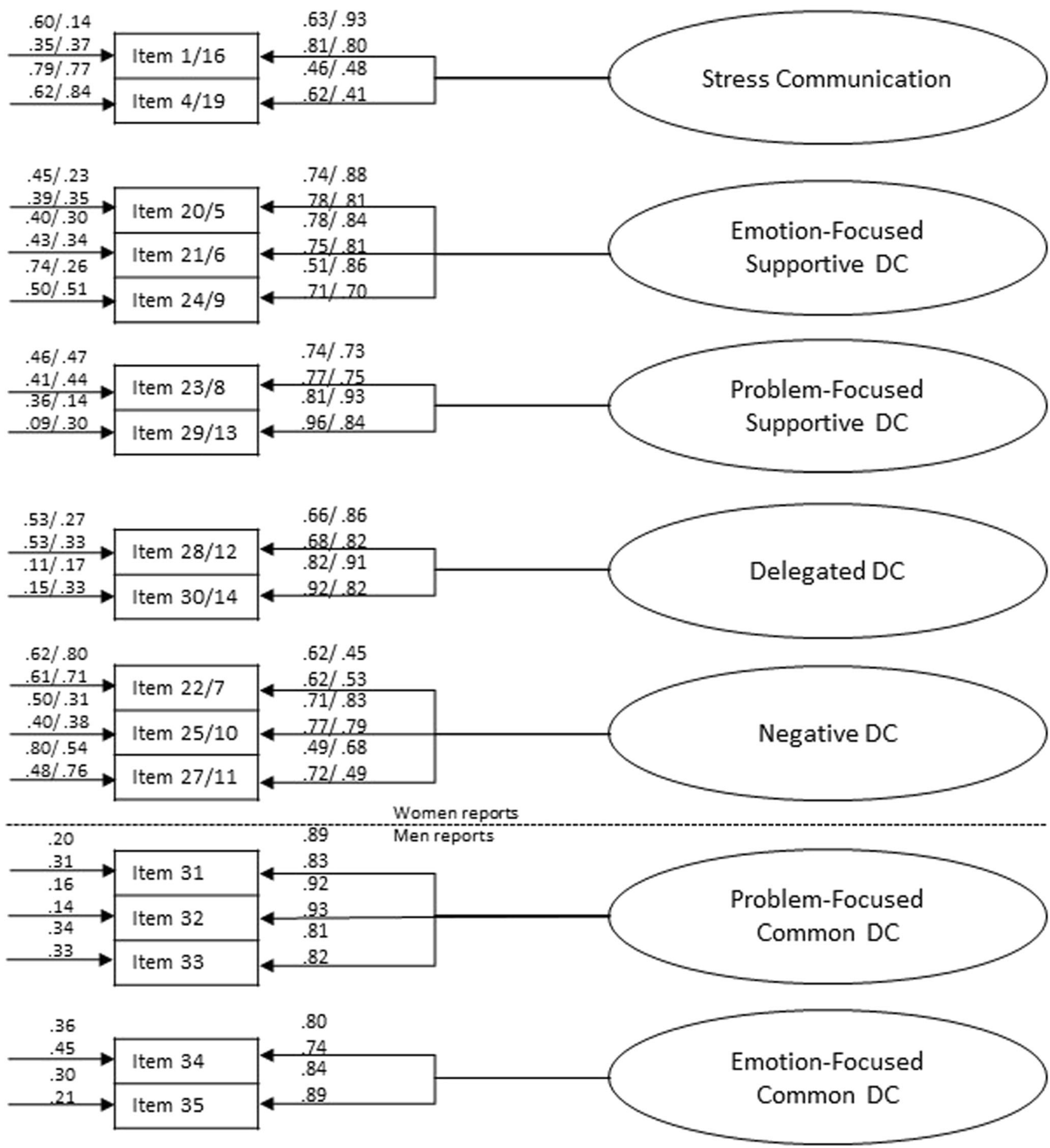

Fig. 1 Confirmative factor analysis for DCI: self, partner and common DC

increasingly more constraints: configural invariance implies that the same factor structure can be established across groups; metric invariance confirms the same factor structure and factor loadings across groups; scalar invariance confirms the same factor structure, factor loadings, and intercepts across groups, and residual invariance confirms the same factor structure, factor loadings, intercepts, and residual variances across groups. It was assumed that because a large-sample chi-square test detects even very small CFI changes, a change in CFI between models of less than 0.01 means the existence 
Table 3 Descriptive statistics, internal consistency and the gender differences test

\begin{tabular}{|c|c|c|c|c|c|c|c|c|c|c|}
\hline & \multicolumn{3}{|l|}{ Men } & \multicolumn{3}{|l|}{ Women } & \multicolumn{3}{|c|}{ Men \& Women } & \multirow[b]{2}{*}{$t$} \\
\hline & $M$ & $S D$ & $\alpha$ & $M$ & $S D$ & $\alpha$ & $M$ & $S D$ & $\alpha$ & \\
\hline SC by Self & 6.52 & 1.85 & .66 & 7.06 & 1.63 & .45 & 13.16 & 3.02 & .57 & $5.35^{*}$ \\
\hline ESDC by Self & 11.71 & 1.99 & .74 & 12.15 & 1.75 & .68 & 11.93 & 1.88 & .74 & 2.78 \\
\hline PSDC by Self & 7.60 & 1.53 & .80 & 7.43 & 1.54 & .75 & 7.51 & 1.54 & .80 & -1.28 \\
\hline DDC by Self & 7.16 & 1.52 & .78 & 6.56 & 1.66 & .78 & 6.86 & 1.62 & .78 & $-4.42 *$ \\
\hline NDC by Self & 5.32 & 2.30 & .75 & 4.80 & 2.03 & .60 & 6.87 & 2.72 & .69 & -2.25 \\
\hline SC by Partner & 7.21 & 1.66 & .49 & 6.05 & 2.00 & .61 & 13.12 & 3.12 & .60 & $-8.11^{*}$ \\
\hline ESDC by Partner & 11.52 & 2.40 & .86 & 10.96 & 2.88 & .90 & 11.24 & 2.66 & .86 & -2.48 \\
\hline PSDC by Partner & 6.96 & 1.68 & .79 & 7.00 & 1.90 & .80 & 6.98 & 1.79 & .79 & .21 \\
\hline DDC by Partner & 6.10 & 1.78 & .84 & 6.75 & 1.91 & .88 & 6.42 & 1.87 & .84 & $4.13 *$ \\
\hline NDC by Partner & 5.39 & 2.27 & .60 & 5.72 & 2.50 & .69 & 8.42 & 2.74 & .66 & 3.33 \\
\hline ECDC & 6.97 & 1.94 & .79 & 6.72 & 2.19 & .79 & 6.85 & 2.07 & .79 & -1.40 \\
\hline PCDC & 11.80 & 2.28 & .89 & 11.80 & .51 & .90 & 11.78 & 2.40 & .90 & -1.60 \\
\hline DC evaluation & 7.81 & 1.65 & .88 & 7.48 & 1.92 & .92 & 7.65 & 1.80 & .90 & -2.17 \\
\hline DC by Self & 46.09 & 6.13 & .71 & 46.61 & 5.32 & .62 & 46.35 & 5.74 & .67 & 1.05 \\
\hline DC by Partner & 46.76 & 5.98 & .65 & 45.61 & 6.66 & .66 & 46.18 & 6.35 & .65 & -2.12 \\
\hline DCI total & 111.62 & 13.48 & .87 & 110.71 & 13.36 & .90 & 111.17 & 13.41 & .86 & -.79 \\
\hline
\end{tabular}

$* p<0.05$

$D C$ dyadic coping, $S C$ stress communication, $E S D C$ emotion-focused supportive DC, $P S D C$ problem-focused supportive DC, $D D C$ delegated DC, $N D C$ negative DC, $E C D C$ emotion-focused common DC, $P C D C$ problem-focused common DC

of a given invariance, even if $p<0.05$ (Cheung and Rensvold 2002). Analysis of configural invariance across gender indicates good fit indices in DC by Self, by Partner and Common DC scales (Table 6). The metric invariance model is not significant, which supports invariance for all DC scales across gender. The scalar invariance model across gender is significant but the difference in the CFI values between metric and scalar invariance is very slight $(\Delta \mathrm{CFI}<.01)$, which supports full scalar invariance for the Self, Partner, and Common DC subscales. Additionally residual invariance across gender for DC by Partner and Common DC is established. The findings substantiate the assumption that Polish men and women respond to items within subscales in a similar way.

MI analysis across cultures was conducted between Poland and Switzerland (where the DCI was developed) and between Poland and Romania, as both countries belong to the same group of post-communist East-European countries. Good fit indices confirm the configural invariance across Polish-Swiss (Table 7) and Polish-Romanian (Table 8) samples for all the DC scales in men and women. Despite the fact that the configural and metric invariance models are significant, except the DC by Partner scale in men, a change can be observed in CFI of less than 0.01, which substantiates the existence of a metric invariance across Polish-Romanian samples for all the DC scales and for Polish-Swiss samples, except DC by Self in women. Regarding scalar invariance across these three countries, only partial support was found. The findings confirm scalar invariance for men and women in the DC by Partner scale for the Polish-Swiss samples and in the Common DC scale for the Polish-Romanian samples.

\section{Discussion}

The DCI was based on the theoretical systemic-transactional model by Bodenmann (2005). To date, many tests have been carried out to check the psychometric properties of the tool (Bodenmann 1997, 2008; Donato et al. 2009; Ledermann et al. 2010; Vedes et al. 2013; Falconier et al. 2013a, b; Fallahchai et al. 2017; Levesque et al. 2014; Randal et al. 2016; Rusu et al. 2016; Xu et al. 2016), and the studies showed the satisfactory reliability and validity of the Inventory. The aim of this article was to analyze the psychometric properties of the Polish version of the DCI and its validation in a Polish sample. In our study, including 550 subjects aged 20-72 who were in an at least one-year-long heterosexual relationship, the proposed factor structure of DCI was confirmed after removing two pairs of items (2/17, $3 / 18$ ) from the SC scale and one pair of items $(15 / 26)$ from the NDC scale. Testing the $5+2$-factor model, factor analysis shows that most items in the model have satisfactory properties. The internal consistency results of the DCI range from Cronbach's Alpha .70 to .92. The exceptions are the SC and NDC scales, which present lower reliability. This is partly 


|


Table 5 Correlations between DC subscales and the KKMiP and the PBF scales

\begin{tabular}{|c|c|c|c|c|c|c|c|c|c|}
\hline \multirow[t]{2}{*}{ DCI } & \multicolumn{6}{|l|}{ KKMiP } & \multicolumn{3}{|l|}{ PFB } \\
\hline & $\begin{array}{l}\mathrm{S} \\
\text { by Self }\end{array}$ & $\begin{array}{l}\text { I } \\
\text { by Self }\end{array}$ & $\begin{array}{l}\mathrm{D} \\
\text { by Self }\end{array}$ & $\begin{array}{l}\mathrm{S} \\
\text { by Partner }\end{array}$ & $\begin{array}{l}\text { I } \\
\text { by Partner }\end{array}$ & $\begin{array}{l}\mathrm{D} \\
\text { by Partner }\end{array}$ & $\mathrm{C}$ & $\mathrm{T}$ & Q \\
\hline \multirow[t]{2}{*}{ SC by Self } & $.45 * *$ & $.48 * *$ & -.03 & $.45^{* *}$ & $.34 * *$ & -.11 & $.43 * *$ & $.45 * *$ & -.10 \\
\hline & $.56^{* *}$ & $.61 * *$ & $-.24 * *$ & $.53 * *$ & $.52 * *$ & $-.26 * *$ & $.58 * *$ & $.54 * *$ & $-.29 * *$ \\
\hline \multirow[t]{2}{*}{ ESDC by Self } & $.60 * *$ & $.56 * *$ & $-.20 * *$ & $.49 * *$ & $.44 * *$ & $-.17 * *$ & $.51 * *$ & $.53 * *$ & $-.18 * *$ \\
\hline & $.70 * *$ & $.58 * *$ & $-.31 * *$ & $.65 * *$ & $.52 * *$ & $-.36 * *$ & $.58 * *$ & $.52 * *$ & $-.38 * *$ \\
\hline \multirow[t]{2}{*}{ PSDC by Self } & $.42 * *$ & $.39 * *$ & .03 & $.34 * *$ & $.27 * *$ & .00 & $.36 * *$ & $.38 * *$ & -.00 \\
\hline & $.54 * *$ & $.41 * *$ & -.03 & $.52 * *$ & $.53 * *$ & $-.14 *$ & $.52 * *$ & $.49 * *$ & $-.22 * *$ \\
\hline \multirow[t]{2}{*}{ DDC by Self } & $.24 * *$ & $.27 * *$ & -.02 & .10 & .03 & .03 & $.13 *$ & .09 & .06 \\
\hline & $.39 * *$ & $.37 * *$ & $-.13 *$ & $.35^{* *}$ & $.31 * *$ & $-.16 * *$ & $.36^{* *}$ & $.30 * *$ & $-.20 * *$ \\
\hline \multirow[t]{2}{*}{ NDC by Self } & $-.30 * *$ & $-.16 * *$ & $.35 * *$ & $-.32 * *$ & $-.25 * *$ & $.38 * *$ & $-.26 * *$ & $-.28 * *$ & $.43 * *$ \\
\hline & $-.38 * *$ & $-.26 * *$ & $.46^{* *}$ & $-.42 * *$ & $-.28 * *$ & $.46^{* *}$ & $-.30 * *$ & $-.29 * *$ & $.46^{* *}$ \\
\hline \multirow[t]{2}{*}{ SC by Partner } & $.34 * *$ & $.35 * *$ & $-.13 *$ & $.41 * *$ & $.52 * *$ & $-.14 *$ & $.55 * *$ & $.46^{* *}$ & -.10 \\
\hline & $.45^{* *}$ & $.42 * *$ & -.09 & $.53 * *$ & $.54 * *$ & $-.15^{*}$ & $.60 * *$ & $.47 * *$ & $-.26 * *$ \\
\hline \multirow[t]{2}{*}{ ESDC by Partner } & $.47 * *$ & $.39 * *$ & $-.21 * *$ & $.70 * *$ & $.63 * *$ & $-.43 * *$ & $.61 * *$ & $.61 * *$ & $-.42 * *$ \\
\hline & $.59 * *$ & $.51 * *$ & $-.27 * *$ & $.71 * *$ & $.63^{* *}$ & $-.40 * *$ & $.64 * *$ & $.59 * *$ & $-.47 * *$ \\
\hline \multirow[t]{2}{*}{ PSDC by Partner } & $.41 * *$ & $.38 * *$ & -.11 & $.57 * *$ & $.47 * *$ & $-.26 * *$ & $.57 * *$ & $.57 * *$ & $-.24 * *$ \\
\hline & $.52 * *$ & $.46^{* *}$ & $-.13 *$ & $.53 * *$ & $.54 * *$ & $-.22 * *$ & $.45^{* *}$ & $.50 * *$ & $-.30 * *$ \\
\hline \multirow[t]{2}{*}{ DDC by Partner } & $.39 * *$ & $.30 * *$ & $-.13 *$ & $.59 * *$ & $.47 * *$ & $-.36 * *$ & $.45 * *$ & $.43 * *$ & $-.32 * *$ \\
\hline & $.39 * *$ & $.38 * *$ & $-.16^{*}$ & $.54 * *$ & $.48 * *$ & $-.31 * *$ & $.41 * *$ & $.39 * *$ & $-.36^{* *}$ \\
\hline \multirow[t]{2}{*}{ NDC by Partner } & $-.26 * *$ & -.11 & $.32 * *$ & $-.47 * *$ & $-.41 * *$ & $.47 * *$ & $-.40 * *$ & $-.37 * *$ & $.48^{* *}$ \\
\hline & $-.32 * *$ & $-.21 * *$ & $.38 * *$ & $-.55^{* *}$ & $-.34 * *$ & $.50 * *$ & $-.36 * *$ & $-.25 * *$ & $.56 * *$ \\
\hline \multirow[t]{2}{*}{ ECDC } & $.53 * *$ & $.55 * *$ & $-.25 * *$ & $.64 * *$ & $.62 * *$ & $-.36 * *$ & $.59 * *$ & $.66 * *$ & $-.36 * *$ \\
\hline & $.55^{* *}$ & $.54 * *$ & $-.15^{*}$ & $.55^{* *}$ & $.55^{* *}$ & $-.30 * *$ & $.58 * *$ & $.60 * *$ & $-.26 * *$ \\
\hline \multirow[t]{2}{*}{ PCDC } & $.50 * *$ & $.35^{* *}$ & $-.13 *$ & $.62 * *$ & $.42 * *$ & $-.23 * *$ & $.58 * *$ & $.52 * *$ & $-.26 * *$ \\
\hline & $.63 * *$ & $.50 * *$ & $-.25 * *$ & $.68 * *$ & $.62 * *$ & $-.34 * *$ & $.66 * *$ & $.59 * *$ & $-.38 * *$ \\
\hline \multirow[t]{2}{*}{ DC evaluation } & $.54 * *$ & $.46^{* *}$ & $-.32 * *$ & $.73 * *$ & $.58 * *$ & $-.452 * *$ & $.64 * *$ & $.63 * *$ & $-.43 * *$ \\
\hline & $.57 * *$ & $.45 * *$ & $-.30 * *$ & $.70 * *$ & $.61 * *$ & $-.48 * *$ & $.67 * *$ & $.61 * *$ & $-.51 * *$ \\
\hline \multirow[t]{2}{*}{ DC by Self } & $.41 * *$ & $.50 * *$ & $.18 * *$ & $.31 * *$ & $.2 * *$ & .09 & $.39 * *$ & $.36 * *$ & $.14 *$ \\
\hline & $.53 * *$ & $.52 * *$ & .01 & $.47 * *$ & $.52 * *$ & -.03 & $.51 * *$ & $.49 * *$ & -.08 \\
\hline \multirow[t]{2}{*}{ DC by Partner } & $.45 * *$ & $.47 * *$ & -.04 & $.56^{* *}$ & $.54 * *$ & $-.17 * *$ & $.60 * *$ & $.56^{* *}$ & $-.13^{*}$ \\
\hline & $.51 * *$ & $.50 * *$ & -.04 & $.55^{* *}$ & $.59 * *$ & -.11 & $.57 * *$ & $.54 * *$ & $-.21 * *$ \\
\hline \multirow[t]{2}{*}{ DCI total } & $.56 * *$ & $.58 * *$ & -.02 & $.61 * *$ & $.55^{* *}$ & $-.15^{*}$ & $.66 * *$ & $.62 * *$ & -0.11 \\
\hline & $.65 * *$ & $.62 * *$ & -.08 & $.65 * *$ & $.68 * *$ & $-.17 * *$ & $.68 * *$ & $.65 * *$ & $-.23 * *$ \\
\hline
\end{tabular}

$* p<.05 ; * * p<.01$; Upper row represents results for women $(n=275)$ and lower row represents results for men $(n=275)$

$D C$ dyadic coping, $S C$ stress communication, ESDC emotion-focused supportive DC, $P S D C$ problem-focused supportive DC, $D D C$ delegated DC, $N D C$ negative DC, $E C D C$ emotion-focused common DC, $P C D C$ problem-focused common DC, $S$ Support, $I$ Involvement, $D$ Depreciation, $C$ communication, $T$ tenderness, $Q$ quarrelling

consistent with the lower Cronbach's alpha results for the scales mentioned in the previous validation studies. Similar results were obtained in the American, French, Persian, Japanese and Latino DCI versions (Ledermann et al. 2010; Falconier et al. 2013a, b; Fallahchai et al. 2017; Randal et al. 2016; Yokotani and Kurosawa 2015), while the reliability of the SC scale turned out to be also weaker in the French, American, Portuguese, Latin and English DCI versions (Ledermann et al. 2010; Falconier et al. 2013a, b; Randal et al. 2016; Vedes et al. 2013). In most of these validation studies, the reliability of the subscales was lower after 3 pairs of items were removed: $2 / 17,3 / 18$ and 15/26. The alpha coefficient for the entire scale was .86 , which confirms the good reliability of the Polish version of the DCI. Gender differences were observed regarding the SC and DDC scales by Self and by Partner. It seems that Polish women present significantly higher scores than men and so their behavior is also evaluated by their partners, which partly corresponds with the results in 
Table 6 Measurement Invariance across gender for Polish sample

\begin{tabular}{|c|c|c|c|c|c|c|c|c|c|}
\hline & \multicolumn{4}{|c|}{ Goodness-of-fit indices } & \multicolumn{5}{|c|}{ Comparison of nested models } \\
\hline & $x^{2}$ & $d f$ & CFI & RMSEA & $\Delta \times 2$ & $\Delta d f$ & $\Delta \mathrm{CFI}$ & $\triangle$ RMSEA & $p$ \\
\hline \multicolumn{10}{|l|}{ DC by Self } \\
\hline Configural & 237.35 & 88 & .931 & .079 & 9.14 & & & & \\
\hline Metric & 246.49 & 95 & .930 & .076 & 26.47 & 7 & .001 & .002 & .243 \\
\hline Scalar & 272.96 & 102 & .921 & .078 & 43.15 & 7 & .009 & .002 & $<.001$ \\
\hline Residual & 316.12 & 114 & .907 & .080 & 46.88 & 12 & .014 & .002 & $<.001$ \\
\hline \multicolumn{10}{|l|}{ DC by Partner } \\
\hline Configural & 208.96 & 88 & .958 & .071 & & & & & \\
\hline Metric & 218.76 & 95 & .957 & .069 & 9.80 & 7 & .001 & .002 & .200 \\
\hline Scalar & 238.86 & 102 & .953 & .070 & 20.10 & 7 & .005 & .001 & $<.01$ \\
\hline Residual & 273.23 & 114 & .945 & .071 & 34.37 & 12 & .008 & .001 & $<.001$ \\
\hline \multicolumn{10}{|l|}{ Common DC } \\
\hline Configural & 20.14 & 8 & .992 & .074 & & & & & \\
\hline Metric & 2.04 & 11 & .994 & .058 & .90 & 3 & .001 & .006 & .826 \\
\hline Scalar & 24.50 & 14 & .993 & .052 & 3.46 & 3 & $<.001$ & .006 & .326 \\
\hline Residual & 36.86 & 19 & .989 & .058 & 12.36 & 5 & .005 & .006 & .030 \\
\hline
\end{tabular}

$D C$ dyadic coping

Latin and Romanian samples (Falconier et al. 2013a, b; Rusu et al. 2016). Women may cope better in both discerning their emotions and in verbalizing what they feel (Hyde and Linn 1988; Nęcki 1996; Oppermann and Weber 2000; Kornaszewska-Polak 2013). Additionally, Polish men significantly more often provide DDC, unlike in other research (e.g. Vedes et al. 2013), which is also confirmed in their partners' evaluations. The Polish family model is characterized as traditional and patriarchal and is based on the roles of an independent, self-sufficient man and a dependent woman (Bednarski 2008; Swedrak 2007). The results confirm that women communicate their needs and ask for help, but men relieve women and take over their responsibilities, which seems to be a partial departure from the stereotypical model of a man who is focused only on financial issues. This might also be related to the fact that the majority of our sample was young people ( $53 \%$ below 40 years of age) who have taken on Western partner models of close relationships.

Correlations between the DCI scales confirm strong relationships with the theoretical construct, except for DDC by Self in women, which does not seem to correlate significantly with the SC by Self scale, ESDC and PSDC by Self and Partner and NDC by Self and Partner, and emotion- and problem-focused CDC. Similar results were achieved in some studies (e.g., Ledermann et al. 2010), although regarding DDC by Partner in women and DDC by Self and by Partner in men these correlations proved to be moderately strong and significant, as in the Portuguese (Vedes et al. 2013) or American samples (Randal et al. 2016). We found other gender differences in dyadic coping in the Polish sample in that PSDC by Self and by Partner are insignificantly related to
NDC by Self and by Partner in women and only with NDC by Self in men.

Dyadic coping strongly correlated with the quality of relational satisfaction and partner communication, which is consistent with previous studies (Bodenmann 2008; Falconier et al. 2015; Ledermann et al. 2010; Vedes et al. 2013; Ruffieux et al. 2014). This confirms the convergent validity of the DCI, which allows indirect inference regarding related concepts based on the results of the test (Hornowska 2007). The strongest significant correlation can be observed between ESDC by Partner and perceived partner's support (S), ECDC and tenderness $(\mathrm{T})$ in both men and women, and between PCDC and communication (C) in men. The results suggest that the use of positive dyadic coping (supportive, delegated, common and stress communication) translates into higher quality and relational satisfaction (Bodenmann 2005; Bodenmann et al. 2011; Levesque et al. 2014; Vedes et al. 2013).

\section{Limitations}

The limitations of the study must be addressed. First of all, due to the fact that a national convenience sample was used, based mostly on well-educated heterosexual couples with high income, all generalizations must be treated with caution. Future research should also include same-sex couples. Secondly, it should be noted that the validation study was carried out on people residing in Poland, so researchers should be careful when using the Polish version of the DCI on Polishspeaking populations living abroad. Thirdly, SC and NDC 
Table 7 Measurement Invariance across cultures for Polish and Swiss samples

\begin{tabular}{|c|c|c|c|c|c|c|c|c|c|}
\hline & \multicolumn{4}{|c|}{ Goodness-of-fit indices } & \multicolumn{5}{|c|}{ Comparison of nested models } \\
\hline & $x^{2}$ & $d f$ & CFI & RMSEA & $\Delta \chi^{2}$ & $\Delta d f$ & $\Delta \mathrm{CFI}$ & $\triangle$ RMSEA & $p$ \\
\hline \multicolumn{10}{|l|}{ DC by Self } \\
\hline \multirow[t]{2}{*}{ Configural } & 248.36 & 88 & .917 & .075 & & 7 & & & .106 \\
\hline & 177.64 & 88 & .962 & .056 & & 7 & & & .839 \\
\hline \multirow[t]{2}{*}{ Metric } & 260.21 & 95 & .914 & .074 & 11.85 & 7 & .018 & .002 & $<.001$ \\
\hline & 181.09 & 95 & .964 & .053 & 3.46 & 7 & .001 & .003 & $<.001$ \\
\hline \multirow[t]{2}{*}{ Scalar } & 301.49 & 102 & .897 & .078 & 41.28 & 12 & .46 & .004 & $<.001$ \\
\hline & 219.04 & 102 & .951 & .060 & 37.95 & 12 & .013 & .007 & $<.001$ \\
\hline \multicolumn{10}{|l|}{ DC by Partner } \\
\hline \multirow{2}{*}{ Configural } & 175.57 & 88 & .975 & .056 & & 7 & & & $<.001$ \\
\hline & 187.64 & 88 & .960 & .059 & & 7 & & & $<.05$ \\
\hline \multirow[t]{2}{*}{ Metric } & 203.57 & 95 & .969 & .060 & 28.01 & 7 & .006 & .004 & $<.001$ \\
\hline & 203.16 & 95 & .957 & .060 & 15.52 & 7 & .003 & $<.001$ & .405 \\
\hline \multirow[t]{2}{*}{ Scalar } & 243.09 & 102 & .959 & .066 & 39.52 & 12 & .009 & .006 & $<.001$ \\
\hline & 310.37 & 102 & .957 & .058 & 7.23 & 12 & $<.001$ & .002 & $<.001$ \\
\hline \multicolumn{10}{|l|}{ Common DC } \\
\hline \multirow[t]{2}{*}{ Configural } & 15.98 & 8 & .995 & .056 & & 3 & & & $<.05$ \\
\hline & 15.38 & 8 & .995 & .054 & & 3 & & & .421 \\
\hline \multirow[t]{2}{*}{ Metric } & 26.82 & 11 & .990 & .067 & 10.85 & 3 & .005 & .011 & $<.001$ \\
\hline & 18.19 & 11 & .995 & .045 & 2.82 & 3 & $<.001$ & .008 & $<.001$ \\
\hline \multirow[t]{2}{*}{ Scalar } & 55.75 & 14 & .974 & .096 & 28.93 & 5 & .016 & .029 & .297 \\
\hline & 40.58 & 14 & .981 & .077 & 22.39 & 5 & .014 & .032 & $<.001$ \\
\hline
\end{tabular}

Upper row and lower row represent the results for women and men, respectively

$D C$ dyadic coping

Table 8 Measurement Invariance across cultures for Polish and Romanian samples

\begin{tabular}{|c|c|c|c|c|c|c|c|c|c|}
\hline & \multicolumn{4}{|c|}{ Goodness-of-fit indices } & \multicolumn{5}{|c|}{ Comparison of nested models } \\
\hline & $x^{2}$ & $d f$ & CFI & RMSEA & $\Delta \chi^{2}$ & $\Delta d f$ & $\Delta \mathrm{CFI}$ & $\triangle \mathrm{RMSEA}$ & $p$ \\
\hline \multicolumn{10}{|l|}{ DC by Self } \\
\hline \multirow[t]{2}{*}{ Configural } & 257.44 & 88 & .938 & .070 & & 7 & & & $<.01$ \\
\hline & 258.56 & 88 & .953 & .070 & & 7 & & & $<.05$ \\
\hline \multirow[t]{2}{*}{ Metric } & 278.24 & 95 & .933 & .070 & 20.80 & 7 & .005 & $<.001$ & $<.001$ \\
\hline & 275.76 & 95 & .950 & .070 & 17.20 & & .003 & .001 & $<.001$ \\
\hline \multirow[t]{2}{*}{ Scalar } & 359.17 & 102 & .906 & .080 & 80.93 & 12 & .027 & .010 & $<.001$ \\
\hline & 429.30 & 102 & .909 & .091 & 153.53 & 12 & .014 & .021 & $<.001$ \\
\hline \multicolumn{10}{|l|}{ DC by Partner } \\
\hline \multirow[t]{2}{*}{ Configural } & 218.17 & 88 & .972 & .062 & & 7 & & & $<.05$ \\
\hline & 288.71 & 88 & .944 & .076 & & 7 & & & $<.001$ \\
\hline \multirow[t]{2}{*}{ Metric } & 234.97 & 95 & .970 & .061 & 16.80 & 7 & .002 & $<.001$ & $<.001$ \\
\hline & 315.54 & 95 & .938 & .077 & 26.83 & 7 & .006 & .001 & $<.001$ \\
\hline \multirow[t]{2}{*}{ Scalar } & 326.95 & 102 & .951 & .075 & 91.98 & 12 & .018 & .014 & $<.001$ \\
\hline & 360.64 & 102 & .927 & .081 & 45.10 & 12 & .011 & .003 & $<.001$ \\
\hline \multicolumn{10}{|l|}{ Common DC } \\
\hline \multirow[t]{2}{*}{ Configural } & 53.13 & 8 & .983 & .120 & & 3 & & & .079 \\
\hline & 35.11 & 8 & .990 & .093 & & 3 & & & 0.285 \\
\hline \multirow[t]{2}{*}{ Metric } & 59.90 & 11 & .981 & .106 & 6.775 & 3 & .001 & .013 & $<.01$ \\
\hline & 38.90 & 11 & .989 & .080 & 3.79 & 3 & $<.001$ & .013 & $<.05$ \\
\hline \multirow[t]{2}{*}{ Scalar } & 71.39 & 14 & .979 & .102 & 11.481 & 5 & .003 & .004 & $<.001$ \\
\hline & 49.14 & 14 & .987 & .080 & 10.24 & 5 & .003 & $<.001$ & $<.01$ \\
\hline
\end{tabular}

Upper row and lower row represent the results for women and men, respectively $D C$ dyadic coping 
scales presented lower reliability, which should be further examined in future studies. Fourth, the study was limited to the psychometric examination of the DCI. It will also be necessary to perform further longitudinal studies using the Inventory in order to carry out an analysis of test-retest reliability and the discriminatory and predictive validity of the DCI.

\section{Conclusions}

Despite these limitations, the obtained results, which are based on confirmatory factor analysis, internal consistency, convergent validity and MI analysis, allow the conclusion that the Polish version of the DCI is a reliable and valid tool that can be used in scientific research as well as in clinical diagnostics, in research both on couples and on individuals in romantic relationships.

The DCI (Bodenmann 2008) is a useful measure for relationship researchers and mental health professionals who work with couples trying to manage stress in their relationships. The current study demonstrates that the Polish version of the DCI is a valid and reliable instrument. Such results serve to provide researchers and clinicians with a useful measure of dyadic coping that is available in the Polish language, which may encourage the continued empirical investigation of the role of dyadic coping in explaining couple functioning in Poland and in cross-cultural contexts. Given the unique role of dyadic coping in intimate relationships and communication processes, it seems that achieving a better understanding of couples' coping behaviors is of great importance. A comprehensive approach to the mechanisms that control the process of dyadic coping is necessary for a better understanding of functioning in close relationships (Bodenmann 2005; Randall and Bodenmann 2017). Subsequent studies of dyadic coping processes can provide additional information on various types of stress (intra- and extra- relational stress, chronic stress, direct and indirect stress, etc.) and their impact on relationships, types of close relationships, and gender or intercultural differences and can also be used as a basis for forming preventive programs that work, particularly with couples experiencing significant levels of stress.

Acknowledgments All procedures performed in studies involving human participants were in accordance with the ethical standards of the institutional research committee (the Ethics Committee at the Institute of Psychology, Jagiellonian University; KE/01/102018) and with the 1964 Helsinki declaration and its later amendments or comparable ethical standards.

Funding Information The author(s) received no financial support for the research, authorship, and/or publication of this article.

The data from the Romanian sample was part of a larger research project coordinated by Dr. Petruta Rusu.
The data from the Swiss sample was part of a research project funded by the Swiss National Science Foundation (SNF: CRSI11_133004/1), awarded to Guy Bodenmann, Veronika Brandstätter, Mike Martin, Fridtjof W. Nussbeck, and Tom Bradbury.

\section{Compliance with Ethical Standards}

Declaration of Conflicting Interests The author(s) declared no potential conflicts of interest with respect to the research, authorship, and/or publication of this article.

Informed Consent Informed consent was obtained from all individual participants included in the study.

Open Access This article is licensed under a Creative Commons Attribution 4.0 International License, which permits use, sharing, adaptation, distribution and reproduction in any medium or format, as long as you give appropriate credit to the original author(s) and the source, provide a link to the Creative Commons licence, and indicate if changes were made. The images or other third party material in this article are included in the article's Creative Commons licence, unless indicated otherwise in a credit line to the material. If material is not included in the article's Creative Commons licence and your intended use is not permitted by statutory regulation or exceeds the permitted use, you will need to obtain permission directly from the copyright holder. To view a copy of this licence, visit http://creativecommons.org/licenses/by/4.0/.

\section{References}

Austin, J. L., \& Falconier, M. K. (2013). Spirituality and common dyadic coping: Protective factors from psychological aggression in Latino immigrant couples. Journal of Family Issues, 34(3), 323-345.

Badr, H., Carmack, C. L., Kashy, D. A., Cristofanilli, M., \& Revenson, T. (2010). Dyadic coping in metastatic breast Cancer. Health Psychology, 29(2), 169-180.

Beach, S. R. H., Lei, M. K., Brody, G. H., Yu, T., \& Philibert, R. A. (2014). Nonsupportive parenting affects telomere length in young adulthood among African Americans: Mediation through substance use. Journal of Family Psychology, 28(6), 967-972.

Beaton, D. E., Bombardier, C., Guillemin, F., \& Ferraz, M. B. (2000). Guidelines for the process of crosscultural adaptation of self-report measures. Spine, 25(24), 3186-3191.

Bednarski, H. (2008). Przemiany struktury i funkcji rodzin polskich w XX I XXI wieku [transformation of the structure and function of Polish families in the XX and XXI century]. Mazowieckie Studia Humanistyczne, 12/1(2), 197-214.

Bodenmann, G. (1995). A systemic-transactional view of stress and coping in couples. Swiss Journal of Psychology, 54, 34-49.

Bodenmann, G. (1997). Dyadic coping - A systemic-transactional view of stress and coping among couples: Theory and empirical findings. European Review of Applied Psychology, 47, 137-140.

Bodenmann, G. (2005). Dyadic coping and its significance for marital functioning. In T. Revenson, K. Kayser, \& G. Bodenmann (Eds.), Couples coping with stress: Emerging perspectives on dyadic coping (pp. 31-50). Washington, DC: American Psychological Association.

Bodenmann, G. (2008). Dyadisches Coping Inventar: Testmanual [dyadic coping inventory: Test manual]. Bern: Huber.

Bodenmann, G., Pihet, S., \& Kayser, K. (2006). The relationship between dyadic coping and marital quality: A 2-year longitudinal study. Journal of Family Psychology, 20(3), 485-493. 
Bodenmann, G., Ledermann, T., \& Bradbury, T. N. (2007). Stress, sex, and satisfaction in marriage. Personal Relationships, 14, 551-569.

Bodenmann, G., Plancherel, B., Beach, S. R. H., Widmer, K., Gabriel, B., Meuwly, N., Charvoz, L., Hautzinger, M., \& Schramm, E. (2008). Effects of coping-oriented couples therapy on depression: A randomized clinical trial. Journal of Consulting and Clinical Psychology, 76(6), 944-954.

Bodenmann, G., Meuwly, N., Bradbury, T., \& Ledermann, T. (2010). Stress, anger, and verbal aggression in intimate relationships: Moderating effects of individual and dyadic coping. Journal of Social and Personal Relationships, 27(3), 408-424.

Bodenmann, G., Meuwly, N., \& Kayser, K. (2011). A comparison of two conceptualizations of dyadic coping and their potential for predicting relationship quality and individual well-being. European Psychologist, 16, 255-266.

Bokhari S.A. (2006). Investigation of the linkage between empathic informal caregiver behaviors, physical symtom experiences, and psychological distress experienced by ovarian cancer patients, Master Thesis, Faculty of Nursing, University of Manitoba, Winnipeg, Manitoba

Campbell, D. T., \& Fiske, D. W. (1959). Convergent and discriminant validation by the multitrait-multimethod matrix. Psychological Bulletin, 56(2), 81-105.

Cheung, G.W., Rensvold, R.B. (2002). Evaluating Goodness-of-Fit Indexes for testing measurement Invariance. Structural Equatation Modeling, 9(2):233-255.

Chow, C. M., Buhrmester, D., \& Tan, C. C. (2014). Interpersonal coping styles and couple relationship quality: Similarity versus complementarity hypotheses. European Journal of Social Psychology, 44, 175186.

Core Team, R. (2016). R: A language and environment for statistical computing. Vienna: R Foundation for Statistical Computing Retrieved from https://www.R-project.org/.

Coyne, J. C., \& Smith, D. A. F. (1991). Couples coping with a myocardial infarction: A contextual perspective on wives' distress. Journal of Personality and Social Psychology, 61, 404-412.

Coyne, J. C., \& Fiske, V. (1992). Couples coping with chronic and catastrophic illness. In T. J. Akamatsu, M. A. P. Stephens, S. E. Hobfoll, \& J. H. Crowther (Eds.), Series in applied psychology: Social issues and questions. Family health psychology (pp. 129-149). Washington, DC, US: Hemisphere Publishing Corp.

DeLongis, A., \& O'Brien, T. (1990). An interpersonal framework for stress and coping: An application to the families of Alzheimer's patients. In M. A. P. Stephens, J. H. Crowther, S. E. Hobfoll, \& D. L. Tennenbaum (Eds.), Stress and coping in later-life families (pp. 221-240). New York: Hemisphere Press.

Donato, S., Iafrate, R., Barni, D., Bertoni, A., Bodenmann, G., \& Gagliardi, S. (2009). Measuring dyadic coping: The factorial structure of Bodenmann's dyadic coping questionnaire in an Italian sample. TPM -testing, psychometrics. Methodology in Applied Psychology, 16(1), 25-47.

Endler, N.S., Parker, J.D.A. (1994). Assessment of multidimensional coping: Task, emotion and avoidance strategies. Psychological Assessment. 6, 50-60.

Falconier, M. K., Nussbeck, F., \& Bodenmann, G. (2013a). Dyadic coping in Latino couples: Validity of the Spanish version of the dyadic coping inventory. Anxiety, Stress, and Coping, 26(4), 447-466.

Falconier, M. K., Nussbeck, F., Bodenmann, G., Schneider, H., \& Bradbury, T. (2013b). Stress from daily hassles in couples: Its effects on intradyadic stress, relationship satisfaction, and physical and psychological well-being. Journal of Marital and Family Therapy, 41(2), 221-235.

Falconier, M. K., Jackson, J. B., Hilpert, P., \& Bodenmann, G. (2015). Dyadic coping and relationship satisfaction: A meta-analysis. Clinical Psychology Review, 42, 28-46.
Falconier, M. K., Randall, A. K., Bodenmann, G. (2016). Couples Coping with Stress: A Cross-Cultural Perspective. New York, NY: Routledge.

Fallahchai, R., Fallahi, M., Chahartangi, S., \& Bodenmann, G. (2017). Psychometric properties and factorial validity of the dyadic coping inventory -the Persian version. Current Psychology, 36(2).

Gabriel, B., Bodenmann, G., Beach, S.R.H. (2016). Gender differences in observed and perceived stress and coping in couples with a depressed partner. Open Journal of Depression. (5): 7-20.

Gudmundsson, E. (2009). Guidelines for translating and adapting psychological instruments. Nordic Psychology, 61(2), 29-45.

Hahlweg, K. (1996). Fragebogen zur Partnerschaftsdiagnostik (FPD) [Partnership questionnaire]. Göttingen: Hogrefe.

Heinrichs, N., Zimmerman, T., Huber, B., Herschbach, P., Russell, D. W., \& Baucom, D. H. (2011). Cancer distress reduction with a couplebased skills training: A randomized controlled trial. Annual Behavioral Medicine, 43(2), 239-252.

Hornowska, E. (2007). Testy psychologiczne : teoria i praktyka [psychological tests: Theory and practice]. Warszawa: Scholar.

Hu, L., \& Bentler, P. M. (1999). Cutoff criteria for fit indexes in covariance structure analysis: Conventional criteria versus new alternatives. Structural Equation Modeling, 6(1), 1-55.

Hyde, J. S., \& Linn, M. C. (1988). Gender differences in verbal ability: A meta-analysis. Psychological Bulletin, 104, 53-69.

Iafrate, R., Bertoni, A., Donato, S., \& Finkenauer, K. (2012). Perceived similarity and understanding in dyadic coping among young and mature couples. Personal Relationships, 19, 401-419.

Janicka, I. (2008). Stosunki partnerskie w związkach niemałżeńskich [Partner relations in non-marital relationships]. Przeglad Psychologiczny, 51(1), 37-53.

Johnson, M. D., Galambos, N. L., Finn, C., \& Neyer, F. J. (2017) Pathways between self-esteem and depression in couples. Developmental Psychology, 53(4), 787-799.

Kardatzke, K.N. (2009). Perceived Stress, Adult Attachment, Dyadic Coping and Marital Satisfaction of Counseling Graduate Students, A Dissertation Thesis, Uncg 0154D 10186.

Kaźmierczak, M., \& Plopa, M. (2012). Kwestionariusz komunikacji matżeńskiej i partnerskiej. Komunikacja $w$ bliskich zwiazkach [Marriage and partner communication questionnaire. Communication in close relationships]. Warszawa: Vizja Press\&IT.

Kornaszewska-Polak, M. (2013). Style komunikowania sie kobiet $i$ mężczyzn a sytuacje trudne [communication styles in women and men and difficult situations]. Kwartalnik Naukowy, 2(13), 102-121.

Lazarus, R. S., \& Folkman, S. (1984). Stress, appraisal, and coping. New York: Springer.

Ledermann, T., Bodenmann, G., Gagliardi, S., Charvoz, L., Verardi, S., Rossier, J., Bertoni, A., \& Iafrate, R. (2010). Psychometrics of the dyadic coping inventory in three language groups. Swiss Journal of Psychology, 69, 201-212.

Levesque, C., Lafontaine, M. F., Caron, A., \& Fitzpatrick, J. (2014). Validation of the English version of the dyadic coping inventory. Measurement and Evaluation in Counseling and Development, 47, 215-225.

Martos, T., Szabo, E., Koren, R., \& Sallay, V. (2019). Dyadic coping in personal projects of romantic partners: Assessment and associations with relationship satisfaction. Current Psychology, 1-4.

Meuwly, N., Bodenmann, G., Germann, J., Bradbury, T. N., Ditzen, B., \& Heinrichs, M. (2012). Dyadic coping, insecure attachment, and cortisol stress recovery following experimentally induced stress. Journal of Family Psychology, 26(6), 937-947.

Nęcki, Z. (1996). Komunikacja międzyludzka [Interpersonal communication]. Krakow: Wydawnictwo Profesjonalnej Szkoły Biznesu.

Oppermann, K., Weber, E. (2000). Język kobiet. Język mężczyzn. Jak porozumieć sie $w$ miejscu pracy [The language of women. The 
language of men. How to communicate in the workplace]. Gdańsk: Gdańskie Wydawnictwo Psychologiczne.

Pearlin L.I., Schooler C. (1978), The structure of coping. Journal of Health and Social Behaviuor, 19, 2-21.

Randall, A. K., Hilpert, P., Jimenez-Arista, L. E., Walsh, K. J., \& Bodenmann, G. (2016). Dyadic coping in the US: Psychometric properties and validity for use of the English version of the dyadic coping inventory. Current Psychology, 35(4), 570-582.

Randall, A. K., \& Bodenmann, G. (2017). Stress and its associations with relationship satisfaction. Current Opinion in Psychology, 13, 96106.

Revenson, T. A., Abraído-Lanza, A. F., Majerovitz, S. D., \& Jordan, C. (2003). Couples coping with chronic illness: What's gender got to do with it? In T. Revenson, K. Kayser, \& G. Bodenmann (Eds.), Couples coping with stress: Emerging perspectives on dyadic coping. Washington, DC: American Psychological Association.

Rosseel, Y. (2012). Lavaan: An R package for structural equation modeling. Journal of Statistical Software, 48(2), 1-36 Retrieved from http://www.jstatsoft.org/v48/i02/.

Rottmann, N., Hansen, D. G., Larsen, P. V., Nicolaisen, A., Flyger, H., Johansen, C., \& Hage-doorn, M. (2015). Dyadic coping within couples dealing with breast cancer: A longitudinal, population-based study. Health Psychology, 34, 486-495.

Ruffieux, M., Nussbeck, F. W., \& Bodenmann, G. (2014). Long-term prediction of relationship satisfaction and stability by stress, coping, communication, and well-being. Journal of Divorce \& Remarriage, 55(6), 485-501.

Rusu, P. P., Hilpert, P., Turliuc, M. N., \& Bodenmann, G. (2016). Dyadic coping in an eastern European context: Validity and measurement invariance of the Romanian version of dyadic coping inventory. Measurement and Evaluation in Counseling and Development, 49(4), 274-285.
Story, L.B., Bradbury, T.N. (2004). Understanding marriage and stress: Essential questions and challenges. Clinical Psychology Review, 23, $1139-1162$.

Swedrak, J. (2007). Model życia wspótczesnej rodziny [A model of modern family life]. Paedagogia Christiana, 2(20), 163-179.

Vandenberg, R. J., \& Lance, C. E. (2000). A review and synthesis of the measurement invariance literature: Suggestions, practices, and recommendations for organizational research. Organizational Research Methods, 3, 4-70. https://doi.org/10.1177/ 109442810031002.

Vaske, I., Thöne, M. F., Kühl, K., Keil, D. C., Schürmann, W., Rief, W., \& Stenzel, N. M. (2015). For better or for worse: A longitudinal study on dyadic coping and quality of life among couples with a partner suffering from COPD. Journal of Behavioral Medicine, 38, 851862.

Vedes, A., Nussbeck, F. W., Bodenmann, G., Lind, W., \& Ferreira, A. (2013). Psychometric properties and validity of the dyadic coping inventory in Portuguese. Swiss Journal of Psychology, 72(3), 149157.

Xu, F., Hilpert, P., Randall, A. K., Quipuning, L., \& Bodenmann, G. (2016). Validation of the dyadic coping inventory with Chinese couples: Factorial structure, measurement invariance, and construct validity. Psychological Assessment, 28, 127-140.

Yokotani, K., \& Kurosawa, T. (2015). A pilot examination of dyadic coping inventory among Japanese married couples. Psychologia, $58,155-164$.

Publisher's note Springer Nature remains neutral with regard to jurisdictional claims in published maps and institutional affiliations. 